\title{
Nonlocal Boundary Conditions Are Applied to the Analysis of Curve Equations
}

\author{
Qingling Wang*, Lingling Fang, \\ Department of Science Education, Jiangxi University of Technology, \\ Nanchang, 330098 \\ China
}

Received: July 10, 2021. Revised: December 5, 2021. Accepted: January 8, 2022. Published: January 10, 2022.

\begin{abstract}
The traditional curve equation solution method has a low accuracy, so the non-local boundary conditions are applied to the curve equation solution. Firstly, the solution coordinate system is established, and then the key parameters are determined to solve the curve equation. Finally, the curve equation is solved by combining the non-local boundary conditions. The experiment proves that the method of this design is more accurate than the traditional method in solving simple curve equation or complex curve equation.
\end{abstract}

Keywords-Non-local boundary condition, curve equation, energy attenuation, initial boundary value problem, high order.

\section{INTRODUCTION}

I $\mathrm{N}$ the cartesian coordinate system, if the point on a curve $\mathrm{C}$ (regarded as a set of points or a trajectory of points suitable for certain conditions) has the following relationship with the real number solution of a binary equation $\mathrm{f}(\mathrm{x}, \mathrm{y})=0$ : (1) The coordinates of points on the curve are all solutions to this equation; (2) The points with the solution of this equation as the coordinate are all points on the curve. So, this equation is called the equation of the curve, and this curve is called the curve of the equation, which is the equation of the curve.

At the moment, the soliton problems appear in a large number of curve equation, and have some common features, which has aroused the interest of many physicists, they hope to use the soliton theory to study the plasma physics, elementary particle physics, and some difficult problems in condensed matter physics, and material nonlinear curve equation under the action of law, etc. From the mathematical point of view, it has been found that there exist soliton solutions in a large class of nonlinear development equations, and these nonlinear development equations with soliton solutions have a series of important and common characteristics. In order to investigate the stability and interaction of solitons, a large number of numerical methods for curve development equations have been developed. It is no exaggeration to say that the research on the solution of curve equation has become a new applied mathematics discipline, which attracts thousands of mathematical workers. Therefore, this research will greatly promote the development of mathematical branches such as differential equation, functional analysis, group theory, homoeotic theory and topology. From this, we can see that, for the development of curve equation, consideration of the following two aspects of the problem is complementary.

On the one hand, under what conditions, the definite solution problems of the curve equation under investigation (including Cauchy problem, various mixed initial side value problems and free boundary problems) have unique global classical solutions. On this basis, the global state of the solution is studied. Under what conditions there is no global classical solution to the problem of definite solution of the curve equation under investigation, but the explosion of solution must occur in finite time. On this basis, the behavior of the solution at the point of the curve equation is investigated in depth, such as whether the solution itself or a partial derivative of the solution first produces the solution parameters, the singularity of the solution at the point of the curve equation and the properties of the point set of the curve equation. The significance of studying these two aspects is obvious. The research on the integral state of the solution of some important mathematical and physical equations (for example, the stability of the solution) and the discussion on the numerical solution methods should take the integral existence of the solution as the premise.

On the other hand, if it is found that the solution will be blown up in a finite time, and the blasting behavior is not allowed by the corresponding physical model, then the mathematical model is wrong and must be modified. If this blasting condition is allowed by the corresponding physical model, the corresponding physical process never ending at a certain moment, must continue to develop, we must in a wider function class inspects the solution of the problems (such as the aerodynamic equations, is considering the possibility of shock wave, and in class containing intermittent function solving). 
Based on the above analysis, some scholars have studied the solution of curve equation. For example, Zhang studies the evaluation application of curve parameter equation [1]; Guo solves the curve differential equation through MATLAB, and explains the error between the approximate differential equation and the accurate differential equation through data and images, so as to improve the accuracy of solution [2]; Gao solved the groundwater infiltration curve. Although the above methods have solved the curve equation, the above methods have the problem of low accuracy [3]. Based on this, this paper applies nonlocal boundary conditions to the solution of curve equations to solve the problem of low accuracy of traditional solutions.

\section{ApPLICATION Of NON-LOCAL BOUNDARY CONDITIONS TO THE CURVE EQUATION}

The boundary conditions with integral operators are non-local boundary conditions, which have a good application background in the theories of thermal reaction, etc. The diffusion equations with non-local boundaries can describe various physical phenomena. For example, chemical engineering, thermal elasticity, population dynamic heat conduction process control theory, medical life science, etc. [1]. In this study, the non-local boundaries involved mainly include the following:

$$
\begin{aligned}
& u(0, t)=\int_{i=1}^{1} 0, u(x, t)=E(t) \\
& \frac{\partial u}{\partial x}(0, t)=(G, t), \int_{i=1}^{1} 0 u(x, t) d x=E(t) \\
& \int_{i=1}^{1} 0 u(x, t) d x=G(t), \int_{i=1}^{1} 0 u(x, t) d x=E(t) \\
& u(x, t)=\int_{i=1}^{1} 0 u(x, t) d x \\
& \frac{\partial u}{\partial u}=\int_{i=1}^{0} i g(x) u
\end{aligned}
$$

The application of non-local boundary conditions in the curve equation is shown in Fig. 1.

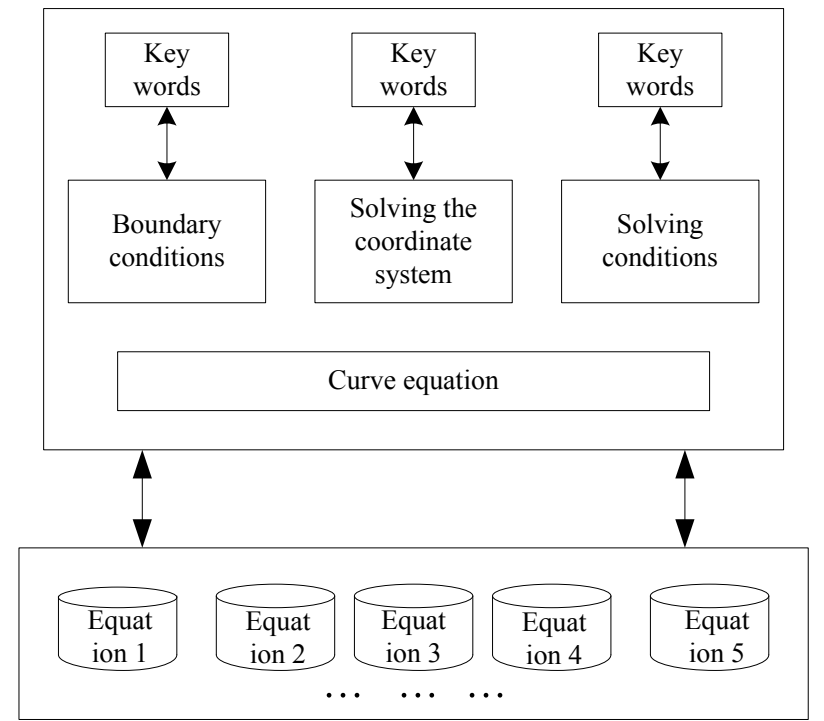

Fig. 1 Application of non-local boundary conditions in curve equation analysis model framework

\section{SOLVE FOR FRAME ESTABLISHMENT}

Consider the system of curves with weighted non-local sources and absorption terms:

$\left\{\begin{array}{l}u_{i}=\Delta x+a(x) \int_{i=1}^{a} d \\ v_{i}=\Delta v+b(x) \\ u(x, t)=v(x, t)=0 \\ u(x, o)=u 0, v o=v(x)\end{array}\right.$

where, $\int_{i=1}^{a} d$ is the bounded region with smooth boundary, and $\Delta x$ is $b(x), a(x), v(x)$, and respectively represent non-negative continuous functions.

The idea of using the cubic design fitting method to fit the curve is as follows:

First, according to the number of unknown parameters to determine the appropriate orthogonal table, and choose the appropriate bit series. For some of the items we can calculate, we can take advantage of the multibit level and increase the appropriate number of rows.

Second: According to the developed parameter bit level table, starting from the initial condition, the center bit level of each parameter is taken as the value of the parameter of the initial condition, and the values of other bit levels are determined according to the ratio or arithmetic sequence [2]-[5].

Third: The developed parameter bit level table and the condition number of the orthogonal table corresponding to each level, the orthogonal table column number corresponding to each parameter, it can form the orthogonal calculation table, and according to the corresponding bit level of each condition level according to the corresponding value of the parameter bit level table, to choose the optimal parameter for the first time.

Fourth: The parameter combination corresponding to each 
condition sign in the orthogonal calculation table is a kind of combination type, according to which we can calculate all the remaining sum of squares under each condition sign;

Fifth: Take the value of the optimal combination parameters obtained in the first round of optimization as the initial value of the second round of optimization parameters, and change their common ratio or tolerance to establish the same parameter bit level table.

Sixth: Using the same orthogonal table, in the second round of optimization, the column Numbers corresponding to the three parameters are postponed by one column.

According to the above method, the calculation parameters are constantly optimized until the remaining sum of squares is smaller and smaller, and finally it is closer and closer to a stable value. On this basis, the solution coordinate system is established, and the solution coordinate system satisfies the following initial conditions:

$u(x, t)=0, v(x)=0, u(x, o)=0$

And non-local boundary conditions:

$$
\left\{\begin{array}{l}
\int_{i=1}^{n} 0 u(x, t) d x=0 \\
\int_{i=1}^{n} 0 x u(x, t) d x=0
\end{array}\right.
$$

The approximate solution is constructed in the form of:

$$
u(x, t) d x=\sum_{j=1} b k i n\left(\frac{y}{i}\right)
$$

where, $\sum_{j=1} b$ is the unknown function.

Since there is a certain correlation between many variables in the curve equation, it is necessary to consider some influence of one variable on another. However, the relationship between them can be divided into two categories, one is that their relationship is definite, that is, there is some kind of functional relationship between them. There is a linear relationship, and there is an uncertain relationship, that is, the relationship between variables and variables cannot be determined by an explicit mathematical expression due to the interference of some other factors:

$$
\left\{\begin{array}{l}
\frac{s}{f s d}+j=\sum_{h} j \\
\int_{i=q}^{a} d / g h \rightarrow j
\end{array}\right.
$$

In the above relation, there are two variables, the change of one variable will change due to the change of the other variable. The variables can be randomly selected, or they can be fixed in advance, without affecting the establishment of the solution coordinate system.

\section{Determine The Key Parameters For Solving The CURVE EQUATION}

The nonlinear terms of the nonlinear parabolic equation (group) can come from the diffusion term, the reaction term, the boundary flow term and the coupling relations formed by them
[6]-[10]. The nonlinear terms may lead to the blasting phenomenon and extinction phenomenon at the finite time of the solution. To solve this problem, the key words of curve equation are determined.

The phenomenon of solving the complete key parameters of the curve equation problem:

$$
\left\{\begin{array}{l}
u i=c v \\
r t(x, y)=0 \\
u(x, o)=f g \geq 0
\end{array}\right.
$$

In addition, the key parameter can also be defined as the solution of a derivative at a finite time. The phenomenon of nonsimultaneous solution means that one component of the solution is solved at a finite time and the other component remains positive until that time. Under certain assumption of initial value, the following can be obtained:

First: If $f g \geq 1$, then the solution is always found simultaneously;

Second: If $f g<1, f g>1$, there is an initial value, so that the phenomenon of different simultaneous solution occurs;

Third: If $f g<1<g$, then the solution is always not at the same time;

In addition, the solution rates under different conditions are obtained:

If only the component occurs $v$, it is near the solution time $t$ , is:

When $p<1, g>1$, then:

$u(0, t)=(y-r) / \frac{t}{T}$

When $p=1=g$, then:

$u(0, t)=\frac{t}{T}^{(p-1)} / f g$

When $p>1=g$, then:

$\left\{\begin{array}{l}u(0, t)=o(y-2) \\ v(0, t)=d(T-t)\end{array}\right.$

Meanwhile, in the solution of the curve equation, there exists the phenomenon of different simultaneous solutions of the equations:

$\left\{\begin{array}{l}u=u_{i} \\ u(o, y)=d f(y) \\ u(1, y)=0 \\ w(x, o)\end{array}\right.$

According to the above calculation:

First: If the correct solution $u_{x}$ does not occur, then $q<1$;

Second: If $0<p, q>1$, there is an initial value, so that simultaneous solution occurs;

Third: If $p>q=(1+q) /(1-q)$, then the solution will always have a different error.

There are some errors in the above calculation. Therefore, the evaluation of the quality of index fitting is as follows: 
First: The difference between the measured value and the real value is approximately zero;

Second, the errors of the measured value of a single variable are independent and independent.

Third, the difference of the real value of the measurement is due to the improper measurement method and accidental errors.

Fourth, the error caused by the selection of measurement method is subject to normal distribution under certain conditions, and the error caused by these reasons does not affect the final result.

When analyzing the existence of a certain relation between the two variables, generally speaking, we need a value that constitutes the pair, which needs to be represented in the cartesian coordinate system. The solution equation is as follows:

$$
\left\{\begin{array}{l}
u_{t}=\int \Delta t-j \\
u=\int v=1 \\
u(x, 0)=\int u_{0}(x)
\end{array}\right.
$$

According to the above calculation, the conditions for the existence of the solution and the solution of errors are obtained, and then the properties of the derivative with respect to the solution with respect to time are obtained. The error phenomena of simultaneous and different solutions may occur, which depends on the selection of the initial value, which plays an important role in describing the solution rate. However, there are other problems in the solution of the curve equation. These phenomena are completely different from the parabolic problem with no variable index, and there are appropriate functions. The calculation equation is as follows:

$$
\left\{\begin{array}{l}
g / i=\frac{s}{g} \\
\int_{i=0}^{s} d<c \\
c g>\frac{o}{p}
\end{array}\right.
$$

Based on the above calculation, the conditions for the existence of global and non-global solutions that depend on or are independent of the initial value are obtained, and it is found that these conditions are related to the initial value. Under the premise of using the classical definition, we further study the asymptotic properties of classification and resolution of the wrong phenomena of solutions with non-standard growth conditions [11]-[14]. First of all, the necessary conditions are proved. If errors occur in the solution and other values remain constant, the calculation index must occur at any initial value. Therefore, it is assumed that:

$$
\left\{\begin{array}{l}
\|\theta\| / \frac{w}{d f} \\
\int_{i=1}^{s} e=f g+\beta+\chi f h+h \\
\prod f+g s e
\end{array}\right.
$$

On the other hand, if sufficient is proved, the following conditions are satisfied:

$$
\prod_{i=q}^{f} w=\frac{e}{f+h+d}
$$

This $\prod_{i=q}^{f} w$ exit is unlikely to result in an error at a finite time. According to the continuity of bounded solutions with respect to initial values, there must be initial values, values close to zero, and values close to 1 . The sets of initial values with this property are all open sets under the solution of curve equation, and the sets obtained are all connected. The region of all variables is a region of coexistence. In fact, smaller initial values or larger initial values, which cause the components to be extinguished separately, and to some extent, there are also initial values that cause the extinction to be simultaneous. From the infinite propagation of the heat equation, for the solution of the curve equation, arbitrarily:

$$
\left\{\begin{array}{l}
\frac{a}{\sum_{i=1} w} * g f h \\
\frac{a}{\sum_{d} s \Rightarrow s}
\end{array}\right.
$$

Then the curve equation has a unique and continuous weak solution which depends on the initial value, and the energy satisfies the exponential decay. For variable index parabolic problems with nonlinear coupling conditions, the singular asymptotic property of the solution is characterized by using multiple index parameters. The quantitative relationship between the odd time and the odd rate is determined. But the rate description depends on the maximum value of the variable index parameter. When errors occur at the same time, the solution results under four situations can be obtained:

$$
\begin{aligned}
& \left\{\begin{array}{l}
y(n)=f+d|f| \\
d[f]=\prod c / h \\
d|g| * \frac{g}{d}=g h+i i
\end{array}\right. \\
& \left\{\begin{array}{l}
f g-h=n n / h \\
\frac{s}{d} / h=e r r \\
f(y)+g=t t
\end{array}\right. \\
& \left\{\begin{array}{l}
t=s / f s \\
h+g=h(d) \\
h+|g|=l s
\end{array}\right. \\
& \left\{\begin{array}{l}
\sum_{d} g=r+f g \\
f=j \longrightarrow r D \\
D=F-H d
\end{array}\right.
\end{aligned}
$$

Steps, prove theorem are used to get the estimate on the time 
derivative, the model describes the solution of the two kinds of curve equation conduction process, for with the nonlocal problems not standard growth conditions for the initial value and variable indicators appropriate assumptions, determine the solution at the same time and not at the same time solving index classification, finally determine the curve equation keywords.

\section{CuRve Equation Calculation based on NON-LOCAL BOUNDARY CONDITIONS}

On the basis of the above calculation, find the optimal solution, use this objective function to track and analyze all the data and all the data that may become the objective function, then compare them, and finally find the optimal point we need. If the initial value is not selected well, the curve will diverge in the iterative process of fitting [15]-[18]. Therefore, the optimal solution is found by the following equation.

$$
\left\{\begin{array}{l}
\frac{s}{f}=f+h g \\
g(n)-d(o)=p \\
f h+h=f^{\prime \prime}
\end{array}\right.
$$

Through the above calculation, it is guaranteed that the solution direction of the system is correct and the step size is appropriate when the residual sum of squares is calculated. At the same time, the initial error requirement is relaxed. However, the algorithm process of this method will be very tedious and cannot get the final answer quickly. On this basis, the problem of fitness of semilinear heat conduction equation with non-local boundary conditions is considered, the unique solution of the equation is obtained, and the equation is transformed into a local initial boundary value problem, and then the non-uniform linear parabolic equation under non-local non-uniform boundary conditions is discussed:

$$
\left\{\begin{array}{l}
u t-(a u x)_{n}+b u=f(x, t, u) \\
u(x, 0)=u 0(x) \\
\int_{i=1}^{\beta} \partial x(u, t)=x c(x, t) s x=0
\end{array}\right.
$$

The upper bound of the curve equation can be estimated during this period by selecting four of the observed values with the same time interval. Whenever possible, find all arrays in these values that satisfy these conditions, and obtain multiple estimates of the parameters, obtain the mathematical expectation of the values of these parameters, and treat them as an unbiased estimate of the parameter Numbers, assuming:

$$
\left\{\begin{array}{l}
d=l / f \\
\sum_{c} f / u t \\
f \cdot x c+g h
\end{array}\right.
$$

The solution to this problem is assumed as follows: In the separable normed linear space, any uniformly bounded linear functional sequence must take weakly convergent subsequence, using the following equation [19]:

$$
\left\{\begin{array}{l}
u t \rightarrow(\text { aux })_{n} \text { at }(0, T) \text { Weak convergence } \\
u(x, 0) \text { at }(0, T) \text { Weak convergence } \\
\int_{i=1}^{\beta} \partial x(u, t) a t(0, T) \text { Weak convergence } \\
x c(x, t) \text { at }(0, T) \text { Weak convergence }
\end{array}\right.
$$

According to the above definition, if the conditions in the solution definition are true, the approximate solution construction method can be obtained [20]:

$\left\|\frac{\partial t}{\partial y}\right\|+\beta\left\|b \frac{d}{n q}\right\| \leq L\|u-v\|$

Assuming the continuous dependence of the solution on the initial value, Gronwall inequality can be used to obtain the following equation:

$$
\|u-c\| \leq\left(\int i-\int n\right) * \frac{A}{D}
$$

By means of cubic spline interpolation function, the expressions of each sub-interval in a given interval are given, and the existence of four parameters in a cubic polynomial is proved.

Because:
$\left\{\begin{array}{l}\partial-x /\|p+k\| \\ \left\|\frac{\mu(\partial-t)}{2}\right\| / \frac{w}{i} \\ \left\|\frac{\partial \wp}{4}\right\| \leq n m\end{array}\right.$

So:

$\partial-x /\|p+k\|+\left\|\frac{\mu(\partial-t)}{2}\right\| / \frac{w}{i}-\left\|\frac{\partial \wp}{4}\right\| \leq \frac{\partial}{b} / \frac{1}{2}$

According to the above derivation, energy satisfies exponential decay.

On this basis, the initial boundary value problem of nonlinear higher order equation is solved as follows:

After satisfying the initial conditions:

$u(x, t)=u(x)=\frac{x}{u}$

And non-local boundary conditions:

$\int_{i=1}^{2} 0(x, t)=0, k=0,1,2, \ldots z m$

The definition of the weak solution and the approximate solution are constructed, and the following conditions are met:

$$
\left\{\begin{array}{l}
\frac{\beta n}{\alpha y}, w_{j}+\frac{w}{\square} \\
u_{n}=(x, v) \\
\frac{\varpi}{\vartheta}=\frac{\varpi v}{\vartheta x}(x, 0)
\end{array}\right.
$$

A class of nonlinear ordinary differential equations is obtained by means of nonlocal boundary conditions. In the 
separable normed linear space, in any uniformly bounded linear functional sequence, the weakly convergent subsequence must be selected. On this basis, the continuous dependence solution of the initial value is obtained:

$$
\begin{aligned}
& E(t) \leq \frac{f}{b} * \sum_{i=1}^{3} v \\
& \text { If: } \begin{array}{l}
\int_{S}^{s i} A * \frac{j}{m} \\
\sum_{n} f / y \\
g=\beta f+u j+i y
\end{array}
\end{aligned}
$$

Then, the curve equation has a unique and continuous weak solution that depends on the initial value, and the energy satisfies exponential decay.

If:

$$
\left\{\begin{array}{l}
\frac{r}{m f}+\frac{d}{f}-h f+\beta \sin u=d(x, y) \\
u(x, 0)=u_{1} x \\
\int_{i=1}^{2} y(x, t)=\int_{n}^{s} d(x, t)
\end{array}\right.
$$

For the above calculation, the following results are obtained: If the optimal solution is not found, then:

$$
\left\{\begin{array}{l}
s=f / \frac{X}{Z} \\
\sum_{c} S / g h=D+s \\
\int_{c}^{a} s=u(y)
\end{array}\right.
$$

In order to obtain the optimal solution, first using the power series of Taylor expansion, the cubic spline interpolation function iteration, to represent each child within a specified range interval on the cubic spline interpolation function, and then use the interpolation condition and first and second order derivative continuity, can find out the function of the relation between the coefficient, finally using the two known boundary conditions, a set of equations is obtained, the equation. The segmentation expression eventually needed can be obtained, are:

$$
\left\{\begin{array}{l}
\frac{d}{f g}=d+s f \\
\sum_{o} s+g=u \\
\frac{s}{d} / g h=u
\end{array}\right.
$$

When solving cubic spline function, we need to know the information of interpolation point and the derivative information of two boundary points, while the solution of cubic spline function needs to know the differential information of two boundary points and the differential information of cubic curve.

Based on this, Pearson chi-square is a research method to analyze the correlation between two variables. If Pearson chi-square test results are meaningful, the null hypothesis cannot be rejected, because the two variables are independent of each other and have no relationship. If the Pearson chi-square test results are important, the original hypothesis is rejected, that is, there is a meaningful correlation between the two variables. Pilsonka square test is affected by the size of sample. If the same sample has different sizes, it may get different results. When the test value is equal to 0 , there is no relationship between the two variables. The closer the absolute value of the detection value is to 1 , the stronger the correlation between the two variables is.

By using this process, the existence of simultaneous solutions to two kinds of parabolic problems with variable indices and the asymptotic properties of odd solutions at different times are obtained, and the non-local boundary conditions of the curve equation are analyzed. In this paper, a polynomial based on cubic spline interpolation method is proposed, which can make every simulation result close to the actual data, with higher precision and smoother curve, thus improving the computational efficiency.

\section{EXPERIMENTAL ANALYSIS}

To validate the design based on the nonlocal boundary conditions of the curve equation of the effectiveness of the method, experiment, and the equations of a first order partial derivatives of solving parameters (1) summed up the following conditions, the solution of the singularity at the point curve equation feature conditions (2) summed up the following conditions and the nature of the curve equation of point set conditions (3) summed up the following conditions and the design method of contrast, contrast four methods to solve application effect, to set up the comparative index for solving accuracy and the equation calculating the total time, divided into divided into simple curve equation and complex equation, four experiments.

\section{A. Experimental Configuration}

Due to the large amount of computation involved in the experiment, computer support is needed. The hardware and software configuration involved is shown in Table I:

Table. I Experimental parameters

\begin{tabular}{ccc}
\hline $\begin{array}{c}\text { Serial } \\
\text { number }\end{array}$ & Name & Parameter \\
\hline 1 & processor & Intel Core i3 3220 \\
2 & monitor & AOC I2369V \\
3 & hard disk & Western Digital 500G \\
4 & Graphics card & Colorful GT730K \\
5 & Motherboard & i945 \\
6 & CPU & $2 \times$ Intel Xoenquad-core \\
7 & RAM & X5530 2.40GHz or more \\
& & TwB $\left(4 \mathrm{G}{ }^{*} 2\right)$ \\
8 & power supply & power supplies, supporting \\
& & $1+1$ redundancy
\end{tabular}




$\begin{array}{cc}9 & \text { Optical drive } \\ 10 & \text { operating } \\ 11 & \text { system } \\ & \text { Main frequency }\end{array}$

11
Main frequency

Awesome

Public

DVD-ROM

Windows Server 2003

Above $3.00 \mathrm{~Hz}$

\section{The}

Datasets

(https://github.com/caesar0301/awesome-public-datasets) randomly selected from a mathematical equation for a number of data in the field of physics, as sample data experiment analysis, the experiment is divided into simple curve equation and the complex equation, large data involved in the experiments, the data was analyzed using data analyzer, as shown in Fig. 2.

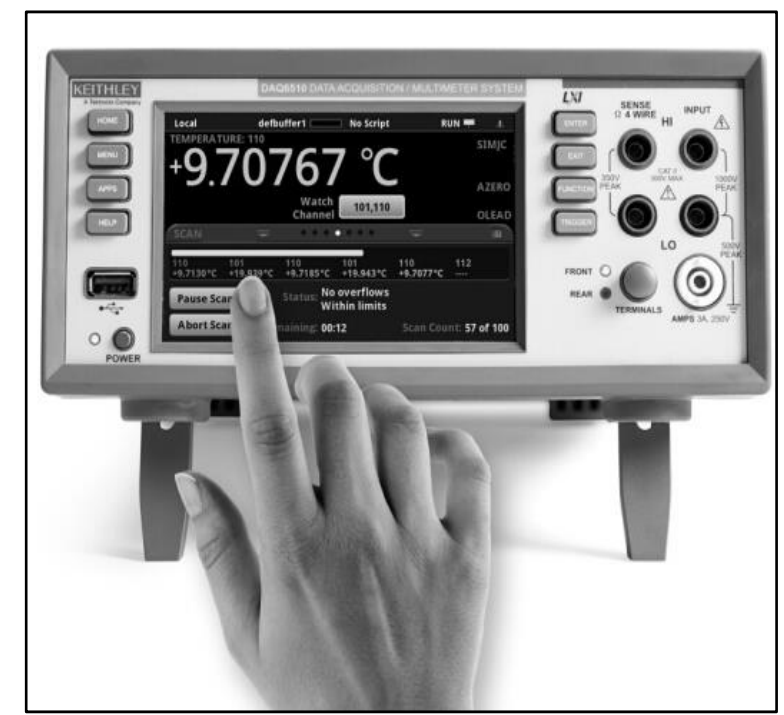

Fig. 2 Experimental data analysis instrument

\section{B. Comparison of Solving Accuracy of Simple Curve Equation}

There is a total of 10 simple equations, and the simple equations can find the specific solution. The specific results are shown in Table II:

Table. II Comparison of solving accuracy of simple curve equation

\begin{tabular}{ccc}
\hline Serial number & Experimental Equation Source & Result \\
\hline 1 & $\begin{array}{l}\text { High School Mathematics } \\
\text { Textbook }\end{array}$ & 12 \\
2 & $\begin{array}{c}\text { High School Mathematics } \\
\text { Textbook }\end{array}$ & 45 \\
3 & $\begin{array}{l}\text { High School Mathematics } \\
\text { Textbook }\end{array}$ & 33 \\
5 & $\begin{array}{l}\text { High School Mathematics } \\
\text { Textbook }\end{array}$ & 45 \\
6 & $\begin{array}{l}\text { High School Mathematics } \\
\text { Textbook }\end{array}$ & 3 \\
7 & $\begin{array}{l}\text { High School Mathematics } \\
\text { Textbook }\end{array}$ & 15 \\
8 & $\begin{array}{l}\text { High School Mathematics } \\
\text { Textbook }\end{array}$ & -45 \\
& $\begin{array}{l}\text { High School Mathematics } \\
\text { Textbook }\end{array}$ & 3
\end{tabular}

9

10
High School Mathematics Textbook

High School Mathematics Textbook
13

132
The method of this design is used to solve the problem respectively with the traditional method, and the accuracy of the two methods is compared. The comparison results are shown in Table III:

Table. III Comparison of solving accuracy of simple curve equation Experimenta Processin Processin Processin The 1 equation $g$ result of $g$ result of $g$ result of results condition condition condition of the $\begin{array}{llll}1 & 2 & 3 & \text { design }\end{array}$ metho

\begin{tabular}{|c|c|c|c|c|}
\hline & & & & $\begin{array}{c}\text { metho } \\
\mathrm{d}\end{array}$ \\
\hline 1 & correct & correct & correct & correct \\
\hline 2 & error & error & error & correct \\
\hline 3 & error & correct & error & correct \\
\hline 4 & error & error & correct & correct \\
\hline 5 & correct & correct & correct & correct \\
\hline 6 & correct & correct & correct & correct \\
\hline 7 & error & error & error & error \\
\hline 8 & correct & error & correct & correct \\
\hline 9 & correct & correct & correct & correct \\
\hline 10 & correct & correct & error & correct \\
\hline
\end{tabular}

It can be seen from Table 3 that only one solution error occurs in the solution of simple curve equation in this design method, while all the other conditions result in four solution errors. Through comparison, it can be seen that the solution method of this design has a high accuracy rate, because the method in this paper not only completes two kinds of parabolic problems with variable indexes, but also analyzes the existence of solutions and the asymptotic properties of odd solutions in different times, and realizes the analysis of non-local boundary conditions of the curve equation.

\section{Comparison of Solving Accuracy of Complex Curve Equation}

The difficulty of the equations involved in this comparison is relatively difficult. 100 equations are randomly selected from the above data set and divided into 5 groups, one group of 20 equations. The accuracy of the two solutions is combined to present the results in the form of a curve. 


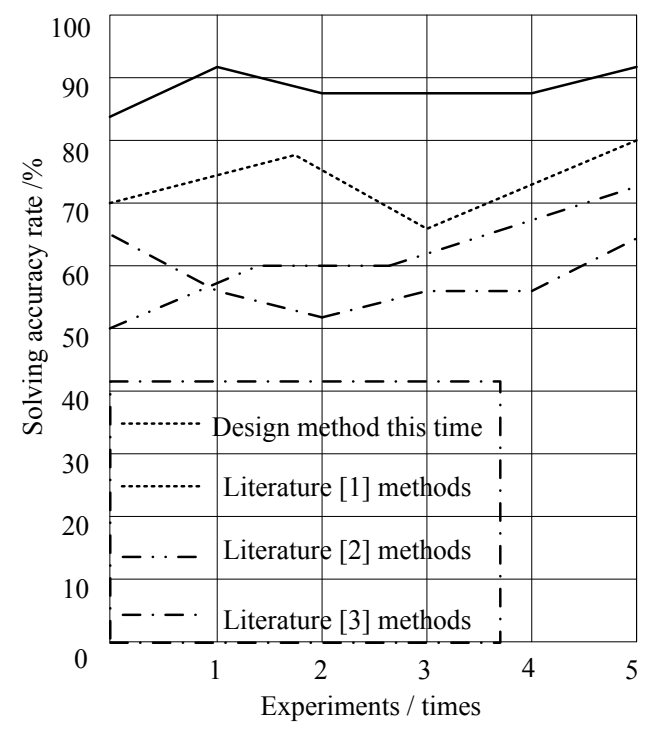

Fig. 3 Comparison results of accuracy of complex curve equation solution

Through the analysis of the comparison results in Fig. 3, it can be seen that this design method has high accuracy in solving 100 equations, while other methods have low accuracy. This is because this design method constructs nonlocal boundary conditions and applies the analysis model to the curve equation, so as to ensure high accuracy.

Therefore, the above experiments can prove that the designed method is more effective and can be applied to the actual curve equation solution.

The time required for the curve equation simulation is the main index to verify the efficiency of the method. The method in this paper is compared with the treatment method under conditions 1,2 and 3, and the experimental results are shown in Fig. 4.

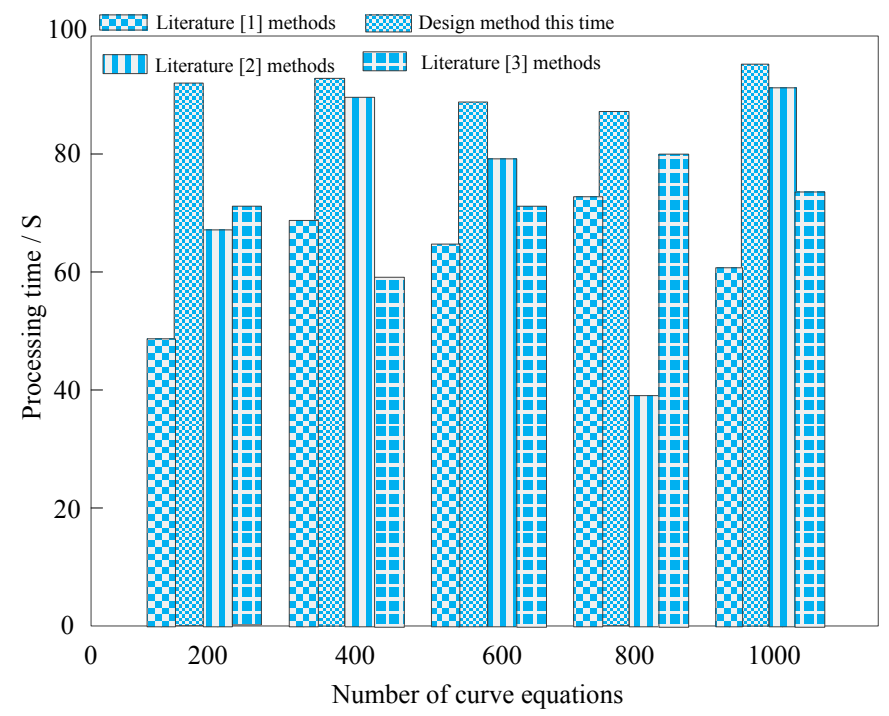

Fig. 4 Comparison of simulation time required by different methods

Under the same equation processing quantity, different processing speeds reflect different processing time. The faster the processing time, the higher the processing efficiency. As shown in Fig. 4, under the limitation of 1000 curve equations, the processing efficiency of this method is generally higher than that of [1], [2] and [3], indicating that the simulation process of this method is faster and the simulation results are more effective, that is, the processing efficiency of this method is higher.

\section{CONCLUSION}

Due to the low accuracy and low efficiency of the traditional method, the application of non-local boundary conditions in the curve equation is analyzed as follows:

(1) After establishing the solution coordinate system, the key parameters of the curve equation are used to solve the solution phenomenon, and the non-local boundary conditions are constructed to apply the analysis model in the curve equation, which has a high computational accuracy, a better fitting effect of the simulation results, and a more effective analysis and prediction of the example, which has a wide range of application value.

(2) Analyzing the conditions for the existence of global and non-global solutions related to the initial value and dependent on or independent of the initial value, and study the asymptotic properties of classification and resolution of the error phenomena of solutions with non-standard growth conditions.

(3) The effectiveness and feasibility of this method are fully demonstrated through comparative tests. It is hoped that it can provide some reference for the related research in this field.

Although the design method can improve the accuracy of curve equation solution, many aspects such as experimental errors will affect the accuracy. Therefore, in the next research process, more factors need to be analyzed to further improve the accuracy of curve equation solution, which will be the future research direction of this paper.

\section{References}

[1] P. Y. Zhang, "Hyperbolic parametric equation with polar angle as parameter," Science and Technology Information, vol. 15, no. 27, pp. 235-236, 2017.

[2] J. Guo, "Error analysis of differential equation of deflection curve of cantilever beam," Shandong Industrial Technology, vol. 284, no. 06, pp. 139-140, 2019.

[3] Y. Gao, "Study on solving groundwater infiltration curve under the combined action of foundation pit dewatering and recharge," Acta Geologica Sinica, vol. 93, no. S1, pp. 127-132, 2019.

[4] F. H. Wen, Y. P. Zhao, M. Z. Zhang, and C. Y. Hu, "Forecasting realized volatility of crude oil futures with equity market uncertainty," Applied Economics, vol. 51, no. 59, pp. 6411-6427, 2019.

[5] M. S. Bhuyan, and M.A. Bakar, "Seasonal variation of heavy metals in water and sediments in the Halda River, Chittagong, Bangladesh," Environmental Science and Pollution Research, vol. 24, no. 35, pp. 27587-27600, 2017. 
[6] J. J. Li, G. H. Wang, and C. Wu, "Characterization of isoprene-derived secondary organic aerosols at a rural site in North China Plain with implications for anthropogenic pollution effects," Scientific Reports, vol. 8, no. 1, pp. 535, 2018.

[7] M. Gong, Z. Cao, and W. Liu, "Supramolecular porphyrin cages assembled at molecular-materials interfaces for electrocatalytic CO reduction," ACS Central Science, vol. 3, no. 9, pp. 1032-1040, 2017.

[8] J. M. Shan, C. Kim, and Z. F. Zhang, "Adsorption of BSA on carbon-coated $\mathrm{Fe} 3 \mathrm{O} 4$ microspheres activated with 1-ethyl-3-(3-dimethylaminopropyl)-carbodiimide hydrochloride," Journal of Wuhan University of Technology (Materials Science Edition), vol. 33, no. 1, pp. $1-8,2018$.

[9] A. J. Torregrosa, A. Broatch, X. Margot, J. García-Tíscar, and R. Cheung, "Local flow measurements in a turbocharger compressor inlet," Experimental Thermal and Fluid Science, vol. 88, pp. 542-553, 2017.

[10]C. J. Carlson, K.R. Burgio, and E.R. Dougherty, "Parasite biodiversity faces extinction and redistribution in a changing climate," Science Advances, vol. 3, no. 9, pp. e1602422, 2017.

[11]F. Schocker, A. Scharf, S. Kull, and U. Jappe, "Detection of the peanut allergens ara $\mathrm{h} 2$ and ara $\mathrm{h} 6$ in human breast milk: Development of 2 sensitive and specific sandwich ELISA assays," International Archives of Allergy and Immunology, vol. 174, no. 1, pp. 17-25, 2017.

[12]Z. He, F. Zhou, X. Xia, F. Wen, and Y. Huang, "Interaction between oil price and investor sentiment: nonlinear causality, time-varying influence, and asymmetric effect," Emerging Markets Finance and Trade, vol. 55, no. 12, pp. 2756-2773, 2019.

[13] S. H. Pishgar-Komleh, A. Akram, and A. Keyhani, "Life cycle energy use, costs, and greenhouse gas emission of broiler farms in different production systems in Iran-a case study of Alborz province," Environmental Science and Pollution Research, vol. 24, no. 69, pp. 1-9, 2017.

[14] R. Mendis, M. Nagai, and W. Zhang, "Artificial dielectric polarizing-beamsplitter and isolator for the terahertz region," Scientific Reports, vol. 7, no. 1, pp. 5909, 2017.

[15]O. A. Mehmet, and M. A. NalanKozac1, "Utility of biochemical markers and RVD/LVD ratio in acute pulmonary embolism risk classification in emergency department," European Review for Medical and Pharmacological Sciences, vol. 21, no. 19, pp. 4391-4397, 2017.

[16] R. Zhou, M. Hirata, and T. Wu, "Spin susceptibility of charge ordered $\mathrm{YBa} 2 \mathrm{Cu} 3 \mathrm{Oy}$ across the upper critical field," Proceedings of the National Academy of Sciences of the United States of America, vol. 114, no. 50, pp. 13148-13153, 2017.

[17] A. S. Laganà, S. G. Vitale, and H. B. Frangež, "Vitamin D in human reproduction: The more, the better? An evidence-based critical appraisal," European Review for Medical and Pharmacological Sciences, vol. 21, no. 18, pp. 4243-4251, 2017.

[18]N. Mokni-Baizig, Y. Gorgi, and M. Elghourabi, "HLA-A*26-A"30 and HLA-DRB1 ${ }^{*} 10$ could be predictors of nasopharyngeal carcinoma risk in high-risk Tunisian families," Journal of Oral Science, vol. 59, no. 2, pp. 289-296, 2017.

[19] B. Ouyang, and Y. Liu, "Lower bound of blasting time for nonlinear nonlocal porous media equation under nonlinear boundary conditions," Journal of Sun Yat sen University (Natural Science Edition), vol. 269, no. 03, pp. 165-170, 2020.

[20] H. Yang, and Y. Zhang, "Approximate controllability of a class of fractional evolution equations with nonlocal integral boundary conditions," Journal of Northwest Normal University: Natural Science Edition, vol. 56, no. 4, pp. 1-7, 2020.

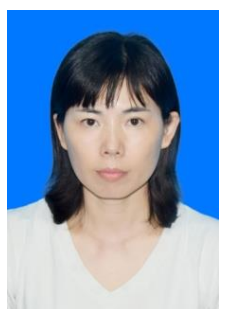

Qingling Wang, female, was born in June 1980. Her professional title is associate professor. In 2003, she won a bachelor's degree in mathematics from Hubei University. In 2006, she won a master's degree in mathematics from Hubei University. She is now working in Jiangxi University of Technology. Her research interests include probability theory and mathematical statistics, education management. She published seven academic papers and participated in four research projects.

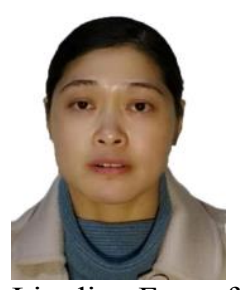

Lingling Fang, female, was born in December 1979. Her title is associate professor. In 2003, she received a bachelor's degree in mathematics and applied mathematics from Jiangxi Normal University. In 2008, she received a master's degree in basic mathematics from Nanjing Normal University. She is now working in Jiangxi University of technology. Her research fields include mathematics and applied mathematics education and mathematical education research. She has published eight academic papers and participated in three research projects.

\section{Author Contributions:}

Qingling Wang determined the key parameters to solve the curve equation. Lingling Fang solves the curve equation with nonlocal boundary conditions.

\section{Creative Commons Attribution License 4.0 (Attribution 4.0 International, CC BY 4.0)}

This article is published under the terms of the Creative Commons Attribution License 4.0

https://creativecommons.org/licenses/by/4.0/deed.en_US 